\title{
Some results on a finite family of Bregman quasi-strict pseudo-contractions
}

\author{
Zi-Ming Wang ${ }^{\mathrm{a}, *}$, Airong Weib \\ ${ }^{a}$ Department of Foundation, Shandong Yingcai University, Jinan, China. \\ ${ }^{b}$ School of Control Science and Engineering, Shandong University, Jinan, China.
}

Communicated by $\mathrm{X}$. Qin

\begin{abstract}
The aim of this article is to establish a common fixed point theorem for a finite family of Bregman quasi-strict pseudocontractions in a reflexive Banach space. Applications to equilibrium problems, variational inequality problems, and zero point problems are provided. (C)2017 All rights reserved.
\end{abstract}

Keywords: Bregman mapping, generalized projection, variational inequality, reflexivity, hybrid method. 2010 MSC: 65K10, 90C30, 47N10.

\section{Introduction}

In recent years, construction of iterative algorithm for seeking fixed points of nonexpansive mappings and strict pseudo-contractions has been extensively investigated; see $[7,9,10,15,23]$ and the references therein. Hybrid projection technique, which was first introduced by Haugazeau [13], is efficient and powerful for treating convergence analysis of mean valued iterative algorithm; see $[3,4,11,12]$ and the references therein. However, many results were obtained in the framework of Hilbert spaces only. The main difficulties are that many nonexpansive mappings in Hilbert spaces are no longer nonexpansive mappings, for example, metric projections. In this connection, Alber [1] introduced a generalized projection operator in Banach spaces which is an analogue of the metric projection in Hilbert spaces. Since then, many authors obtained strong convergence theorems for nonlinear operators based on the generalized projections in Banach spaces; see [14, 22] and the references therein. Another way is to use the Bregman distance instead of the norm, Bregman (quasi-)nonexpansive mappings instead of the (quasi)nonexpansive mappings and the Bregman projection instead of the metric projection; see [18, 20, 21] and the references therein.

Motivated and inspired by the works going in this directions, we propose a new hybrid Bregman projection iterative algorithm for a finite family of Bregman quasi-strict pseudo-contractions and prove strong convergence results in the framework of reflexive Banach spaces. The results presented in this paper improve or enrich the known corresponding results announced in the literature sources listed in this work.

\footnotetext{
*Corresponding author

Email addresses: wangziming@ymail .com (Zi-Ming Wang), weiairong@sdu.edu.cn (Airong Wei)
} 


\section{Preliminaries}

In this section, we collect some preliminaries and lemmas which will be used to prove our main results. Throughout this paper, $E$ is assumed to be a real reflexive Banach space with norm $\|\cdot\|$ and $E^{*}$ the dual space of $E$. The normalized duality mapping from $E$ to $2^{\mathrm{E}^{*}}$ denoted by $\mathrm{J}$ is defined by

$$
\mathrm{J} x=\left\{\mathrm{f} \in \mathrm{E}^{*}:\langle x, \mathrm{f}\rangle=\|\mathrm{x}\|^{2}=\|\mathrm{f}\|^{2}\right\}, \quad \forall x \in \mathrm{E},
$$

where $\langle\cdot, \cdot\rangle$ denotes the generalized duality pairing between $E$ and $E^{*}$. In this paper, we use $\mathbb{R}$ and $\mathbb{N}$ stand for the sets of real numbers and positive integers, respectively.

Let $f: E \rightarrow(-\infty,+\infty]$ be a proper, convex and lower semi-continuous function. We denote by $\operatorname{dom}(f)$ the domain of $f$, that is, $\operatorname{dom}(f):=\{x \in E: f(x)<+\infty\}$. For any $x \in \operatorname{int}(\operatorname{dom}(f))$ and $y \in E$, the right-hand derivative of $f$ at $x$ in the direction of $y$ is defined by

$$
f^{\circ}(x, y)=\lim _{t \rightarrow 0^{+}} \frac{f(x+t y)-f(x)}{t} .
$$

The function $f$ is said to be Gâteaux differentiable at $x$ if $\lim _{t \rightarrow 0^{+}} \frac{f(x+t y)-f(x)}{t}$ exists for any $y$. In this case, $f^{\circ}(x, y)$ coincides with $\nabla f(x)$, the value of the gradient $\nabla f(x)$ of $f$ at $x$. The function $f$ is called Gâteaux differentiable if it is Gâteaux differentiable for any $x \in \operatorname{int}(\operatorname{dom}(f))$. The function $f$ is said to be Fréchet differentiable at $x$ if limit (2.1) is attained uniformly in $\|y\|=1$. The function $f$ is said to be Fréchet differentiable if it is Fréchet differentiable for any $x \in \operatorname{int}(\operatorname{dom}(f))$. Finally, $f$ is called be uniformly Fréchet differentiable on a subset $C$ of $E$ if limit (2.1) is attained uniformly for $x \in C$ and $\|y\|=1$. It is well-known that if a continuous convex function $f: E \rightarrow \mathbb{R}$ is Gâteaux differentiable, then $\nabla f$ is norm-toweak* continuous. Also, it is known that if $f$ is said to be Fréchet differentiable, then $\nabla f$ is norm-to-norm continuous. The function $f$ is said to be strongly coercive if

$$
\lim _{\left\|x_{n}\right\| \rightarrow \infty} \frac{f\left(x_{n}\right)}{\left\|x_{n}\right\|}=\infty
$$

From Reich and Sabach [16], we see if a function $f: X \rightarrow \mathbb{R}$ is uniformly Fréchet differentiable and bounded on bounded subsets of $E$, then $\nabla f$ is uniformly continuous on bounded subsets of $E$ from the strong topology of $E$ to the strong topology of $E^{*}$.

Let $f: E \rightarrow(-\infty,+\infty]$ be a convex and Gâteaux differentiable function. Then the Bregman distance [8] with respect to $f$ is the function $D_{f}: \operatorname{dom}(f) \times \operatorname{int}(\operatorname{dom}(f)) \rightarrow[0,+\infty)$ defined by

$$
D_{f}(x, y)=f(x)-f(y)-\langle x-y, \nabla f(y)\rangle \text {. }
$$

With the function $f$ we associate the bifunction $V_{f}: E \times E^{*} \rightarrow[0,+\infty)$ defined by

$$
V_{f}\left(x, x^{*}\right)=f(x)-\left\langle x, x^{*}\right\rangle+f^{*}\left(x^{*}\right), \quad \forall x \in E, x^{*} \in E^{*} .
$$

Then $V_{f}$ is nonnegative and

$$
V_{f}\left(x, x^{*}\right)=D_{f}\left(x, \nabla f^{*}\left(x^{*}\right)\right),
$$

for all $x \in E$ and $x^{*} \in E^{*}$. Recall that the Bregman projection [16] of $x \in \operatorname{int}(\operatorname{dom}(f))$ onto the nonempty closed and convex set $C \subset \operatorname{dom}(f)$ is the unique vector $P_{C}^{f}(x) \in C$ satisfying

$$
D_{f}\left(P_{C}^{f}(x), x\right)=\inf \left\{D_{f}(y, x): y \in C\right\} .
$$

It should be observed that if $E$ is a smooth and strictly convex Banach space, setting $f(x)=\|x\|^{2}$ for all $x \in$ $E$, we have $\nabla f(x)=2 J x$ for all $x \in E$. Hence $D_{f}(x, y)$ reduces to the Lyapunov function $\phi(x, y)=\|x\|^{2}-$ $2\langle x, J y\rangle+\|y\|^{2}$ for all $x, y \in E$ and the Bregman projection $P_{C}^{f}(x)$ reduces to the generalized projection $\Pi_{C}(x)$ which is defined by $\Pi_{C}(x)=\arg \min _{y \in C} \phi(y, x)$. If $E$ is a Hilbert space $H$, then $D_{f}(x, y)$ becomes $\phi(x, y)=\|x-y\|^{2}$ for all $x, y \in H$ and the Bregman projection $P_{C}^{f}(x)$ becomes the metric projection $P_{C}(x)$.

Similar to the metric projection in Hilbert space, Bregman projections with respect to totally convex and differentiable functions have variational characterizations. 
Lemma 2.1 ([6]). Suppose that $\mathrm{f}$ is Gâteaux differentiable and totally convex on $\operatorname{int}(\operatorname{dom}(\mathrm{f}))$. Let $\mathrm{x} \in \operatorname{int}(\operatorname{dom}(\mathrm{f}))$ and let $\mathrm{C} \subset \operatorname{int}(\operatorname{dom}(f))$ be a nonempty, closed and convex set. If $\hat{x} \in \mathrm{C}$, then the following conditions are equivalent:

(a) the vector $\hat{x}$ is the Bregman projection of $x$ onto $C$ with respect to $f$, i.e., $\hat{x}=P_{C}^{f}(x)$;

(b) the vector $\hat{x}$ is the unique solution of the variational inequality

$$
\langle\nabla f(x)-\nabla f(\hat{x}), \hat{x}-y\rangle \geqslant 0, \quad \forall y \in C ;
$$

(c) the vector $\hat{x}$ is the unique solution of the inequality

$$
D_{f}(y, \hat{x})+D_{f}(\hat{x}, x) \leqslant D_{f}(y, x), \quad \forall y \in C .
$$

Let $E$ be a Banach space and let $B_{r}:=\{z \in E:\|z\| \leqslant r\}$ for all $r>0$ and $S_{E}=\{x \in E:\|x\|=1\}$. Then a function $f: E \rightarrow \mathbb{R}$ is said to be uniformly convex on bounded subsets of $E$ if $\rho_{r}(t)>0$ for all $r, t>0$, where $\rho_{\mathrm{r}}:[0, \infty) \rightarrow[0, \infty]$ is defined by

$$
\rho_{r}(t):=\inf _{x, y \in B_{r},\|x-y\|=t, \alpha \in(0,1)} \frac{\alpha f(x)+(1-\alpha) f(y)-f(\alpha x+(1-\alpha) y)}{\alpha(1-\alpha)} .
$$

Let $f: E \rightarrow(-\infty,+\infty]$ be a convex and Gâteaux differentiable function. Recall that the function $f$ is called totally convex at a point $x \in \operatorname{int}(\operatorname{dom}(f))$ if its modulus of total convexity at $x$, that is, the function $v_{f}: \operatorname{int}(\operatorname{dom}(f)) \times[0,+\infty) \rightarrow[0,+\infty)$, defined by

$$
v_{f}(x, t):=\inf \left\{D_{f}(y, x): y \in \operatorname{int}(\operatorname{dom}(f)),\|y-x\|=t\right\},
$$

is positive whenever $t>0$. The function $f$ is called totally convex when it is totally convex at every point $x \in \operatorname{int}(\operatorname{dom}(f))$. Moreover, the function $f$ is called totally convex on bounded subset of $E$ if $v_{f}(C, t)>0$ for any bounded subset $C$ of $E$ and for any $t>0$, where the modulus of total convexity of the function $f$ on the set $C$ is the function $v_{f}: \operatorname{int}(\operatorname{dom}(f)) \times[0,+\infty) \rightarrow[0,+\infty)$ defined by

$$
v_{f}(C, t):=\inf \left\{v_{f}(x, t): x \in C \cap \operatorname{int}(\operatorname{dom}(f))\right\}
$$

We remark in passing that $f$ is totally convex on bounded sets if and only if $f$ is uniformly convex on bounded sets.

Recall that the function $f$ is said to be sequentially consistent [6] if for any two sequences $\left\{x_{n}\right\}$ and $\left\{y_{n}\right\}$ in $E$ such that the first one is bounded,

$$
\lim _{n \rightarrow \infty} D_{f}\left(y_{n}, x_{n}\right)=0 \Rightarrow \lim _{n \rightarrow \infty}\left\|y_{n}-x_{n}\right\|=0 .
$$

We have the following conclusions about totally convex functions.

Lemma 2.2 ([5]). The function $f$ is totally convex on bounded sets if and only if the function $f$ is sequentially consistent.

Lemma 2.3 ([17]). Let $\mathrm{f}: \mathrm{E} \rightarrow \mathbb{R}$ be a Gâteaux differentiable and totally convex function. If $\mathrm{x}_{0} \in \mathrm{E}$ and the sequence $\left\{D_{f}\left(x_{n}, x_{0}\right)\right\}$ is bounded, then the sequence $\left\{x_{n}\right\}$ is bounded too.

Lemma 2.4 ([16]). Let $f: X \rightarrow \mathbb{R}$ be a convex function which is bounded on bounded subsets of $E$. Then the following assertions are equivalent:

(a) $f$ is strongly coercive and uniformly convex on bounded subsets of $\mathrm{E}$; 
(b) $\mathrm{f}^{*}$ is Fréchet differentiable and $\nabla \mathrm{f}^{*}$ is uniformly norm-to-norm continuous on bounded subsets of $\operatorname{dom}\left(\mathrm{f}^{*}\right)=$ $\mathrm{E}^{*}$.

Let $x \in \operatorname{int}(\operatorname{dom}(f))$, the subdifferential of $f$ at $x$ is the convex set defined by

$$
\partial f(x)=\left\{x^{*} \in E^{*}: f(x)+\left\langle x^{*}, y-x\right\rangle \leqslant f(y), \quad \forall y \in E\right\} .
$$

The Fenchel conjugate of $f$ is the function $f^{*}: E^{*} \rightarrow(-\infty,+\infty]$ defined by

$$
f^{*}\left(x^{*}\right)=\sup \left\{\left\langle x^{*}, x\right\rangle-f(x): x \in E\right\}, x^{*} \in E^{*} .
$$

The function $f$ is said to be essentially smooth if $\partial f$ is both locally bounded and single-valued on its domain. It is called essentially strictly convex, if $(\partial f)^{-1}$ is locally bounded on its domain and $f$ is strictly convex on every convex subset of $\operatorname{dom}(\partial f)$. $f$ is said to be a Legendre, if it is both essentially smooth and essentially strictly convex. When the subdifferential of $f$ is single-valued, it coincides with the gradient $\partial f=\nabla f$.

We remark that if $E$ is a reflexive Banach space. Then we have the following [2]:

(i) $f$ is essentially smooth if and only if $f^{*}$ is essentially strictly convex;

(ii) $(\partial f)^{-1}=\partial f^{*}$;

(iii) $f$ is Legendre if and only if $f^{*}$ is Legendre;

(iv) if $f$ is Legendre, then $\nabla f$ is bijection satisfying $\nabla f=\left(\nabla f^{*}\right)^{-1}, \operatorname{ran}(\nabla f)=\operatorname{dom}\left(\nabla f^{*}\right)=\operatorname{int}\left(\operatorname{dom}\left(f^{*}\right)\right)$ and $\operatorname{ran}\left(\nabla f^{*}\right)=\operatorname{dom}(\nabla f)=\operatorname{int}(\operatorname{dom}(f))$.

Let $\mathrm{T}: \mathrm{C} \rightarrow \mathrm{C}$ be a mapping. A point $p \in \mathrm{C}$ is said to be an asymptotic fixed point of $\mathrm{T}$ if $\mathrm{C}$ contains a sequence $\left\{x_{n}\right\}$ which converges weakly to $p$ such that $\lim _{n \rightarrow \infty}\left\|x_{n}-T x_{n}\right\|=0$. We denote by $\widehat{F}(T)$ the set of asymptotic fixed points of $T$. A point $p \in C$ is said to be a strong asymptotic fixed point of a mapping $T$ if $C$ contains a sequence $\left\{x_{n}\right\}$ which converges strongly to $p$ such that $\lim _{n \rightarrow \infty}\left\|x_{n}-T x_{n}\right\|=0$. We denote by $\widetilde{F}(T)$ the set of strong asymptotic fixed points of $T$.

Recall the following definitions:

Definition 2.5. Let $C$ be a subset of $E$ and let $T: C \rightarrow C$ be a mapping.

(1) $T$ is said to be Bregman relatively nonexpansive if $\widehat{F}(T)=F(T) \neq \emptyset$ and

$$
D_{f}(p, T x) \leqslant D_{f}(p, x), \quad \forall x \in C, p \in F(T) .
$$

(2) $T$ is said to be Bregman weak relatively nonexpansive if $\widetilde{F}(T)=F(T) \neq \emptyset$ and

$$
D_{f}(p, T x) \leqslant D_{f}(p, x), \quad \forall x \in C, p \in F(T) .
$$

(3) $T$ is said to be Bregman quasi-nonexpansive if $F(T) \neq \emptyset$ and

$$
D_{f}(p, T x) \leqslant D_{f}(p, x), \quad \forall x \in C, p \in F(T) .
$$

(4) $T$ is said to be Bregman quasi-strictly pseudo-contractive [20] if there exists a constant $k \in[0,1)$ and $\mathrm{F}(\mathrm{T}) \neq \emptyset$ such that

$$
D_{f}(p, T x) \leqslant D_{f}(p, x)+k D_{f}(x, T x), \quad \forall x \in C, p \in F(T) .
$$

(5) $T$ is said to be quasi- $\phi$-strictly pseudo-contractive if $F(T) \neq \emptyset$ and there exists a constant $k \in[0,1)$ such that

$$
\phi(p, T x) \leqslant \phi(p, x)+k \phi(x, T x), \quad \forall x \in C, p \in F(T) .
$$

(6) A mapping $T: C \rightarrow C$ is said to be closed if for any sequence $\left\{x_{n}\right\} \subset C$ with $x_{n} \rightarrow x \in C$ and $T x_{n} \rightarrow y \in C$ as $n \rightarrow \infty$, then $T x=y$. 
Remark 2.6. From the above definitions, we have following facts:

(1) Bregman relatively nonexpansive mappings, Bregman weak relatively nonexpansive mappings, Bregman quasi-nonexpansive mappings, and Bregman quasi-strict pseudo-contractions are more general than relatively nonexpansive mappings, relatively weak nonexpansive mappings, hemi-relatively nonexpansive mappings, and quasi- $\phi$-strict pseudo-contractions, respectively.

(2) The class of Bregman quasi-strict pseudo-contractions is more general than the class of Bregman relatively nonexpansive mappings, the class of Bregman weak relatively nonexpansive mappings, and the class of Bregman quasi-nonexpansive mappings.

Next, we give some examples of Bregman quasi-strict pseudo-contractions.

Example 2.7 ([18]). Let $E$ be a real reflexive Banach space, $A: E \rightarrow 2^{E^{*}}$ be a maximal monotone mapping, and $f: E \rightarrow(-\infty,+\infty]$ be a uniformly Fréchet differentiable function and bounded on bounded subsets of $E$ such that $A^{-1}\left(0^{*}\right) \neq \emptyset$, then the resolvent

$$
\operatorname{Res}_{A}^{f}(x)=(\nabla f+A)^{-1} \circ \nabla f(x)
$$

is closed and Bregman relatively nonexpansive from $E$ onto $\operatorname{dom}(A)$, so is a closed Bregman quasi-strict pseudo-contraction.

Example 2.8 ([21]). Let $E$ be a smooth Banach space, and define $f(x)=\|x\|^{2}$ for all $x \in E$. Let $x_{0} \neq 0$ be any element of $E$, the mapping $T: E \rightarrow E$ be defined as follows:

$$
\mathrm{T}(\mathrm{x})= \begin{cases}\left(\frac{1}{2}+\frac{1}{2^{n+1}}\right) x_{0}, & \text { if } x=\left(\frac{1}{2}+\frac{1}{2^{n}}\right) x_{0} \\ -x, & \text { if } x=\left(\frac{1}{2}+\frac{1}{2^{n}}\right) x_{0}\end{cases}
$$

for all $n \geqslant 1$. Then $T$ is a Bregman quasi-strict pseudo-contraction.

Example 2.9 ([20]). Let $E=\mathbb{R}$ and define $T, f:[-1,0] \rightarrow \mathbb{R}$ by $f(x)=x$ and $T x=2 x$ for all $x \in[-1,0]$. Then $T$ is a Bregman quasi-strict pseudo-contraction but not a quasi- $\phi$-strict pseudo-contraction.

Before stating our main results, we also need the following lemmas.

Lemma 2.10 ([20]). Let $\mathrm{f}: \mathrm{E} \rightarrow \mathbb{R}$ be a Legendre function which is uniformly Fréchet differentiable on bounded subsets of $\mathrm{E}$. Let $\mathrm{C}$ be a nonempty, closed, and convex subset of $\mathrm{E}$ and let $\mathrm{T}: \mathrm{C} \rightarrow \mathrm{C}$ be a Bregman quasi-strictly pseudo-contractive mapping with respect to $\mathrm{f}$. Then $\mathrm{F}(\mathrm{T})$ is closed and convex.

Lemma 2.11 ([20]). Let $\mathrm{f}: \mathrm{E} \rightarrow \mathbb{R}$ be a Legendre function which is uniformly Fréchet differentiable on bounded subsets of $\mathrm{E}$. Let $\mathrm{C}$ be a nonempty, closed, and convex subset of $\mathrm{E}$ and let $\mathrm{T}: \mathrm{C} \rightarrow \mathrm{C}$ be a Bregman quasi-strictly pseudo-contractive mapping with respect to $f$. Then, for any $x \in \mathrm{C}, \mathrm{p} \in \mathrm{F}(\mathrm{T})$, and $\mathrm{k} \in[0,1)$ the following holds:

$$
D_{f}(x, T x) \leqslant \frac{1}{1-k}\langle\nabla f(x)-\nabla f(T x), x-p\rangle .
$$

\section{Main results}

In this section, we state and prove our main theorem.

Theorem 3.1. Let $\mathrm{E}$ be a real reflexive Banach space and let $\mathrm{C}$ be a nonempty, closed, and convex subset of $\mathrm{E}$. Let $\mathrm{f}: \mathrm{E} \rightarrow \mathbb{R}$ be a strongly coercive Legendre function which is bounded, uniformly Fréchet differentiable, and totally convex on bounded subsets of $\mathrm{E}$, and let $\mathrm{T}_{i}: \mathrm{C} \rightarrow \mathrm{C}$, where $i=1,2, \ldots, \mathrm{N}$, be a finite family of closed and Bregman quasi- $\mathrm{k}_{i}$-strict pseudo-contractions such that $\mathrm{F}=\bigcap_{i=1}^{N} \mathrm{~F}\left(\mathrm{~T}_{i}\right) \neq \emptyset$. Let $\left\{\mathrm{x}_{\mathrm{n}}\right\}$ be a sequence generated by the following iterative algorithm: 


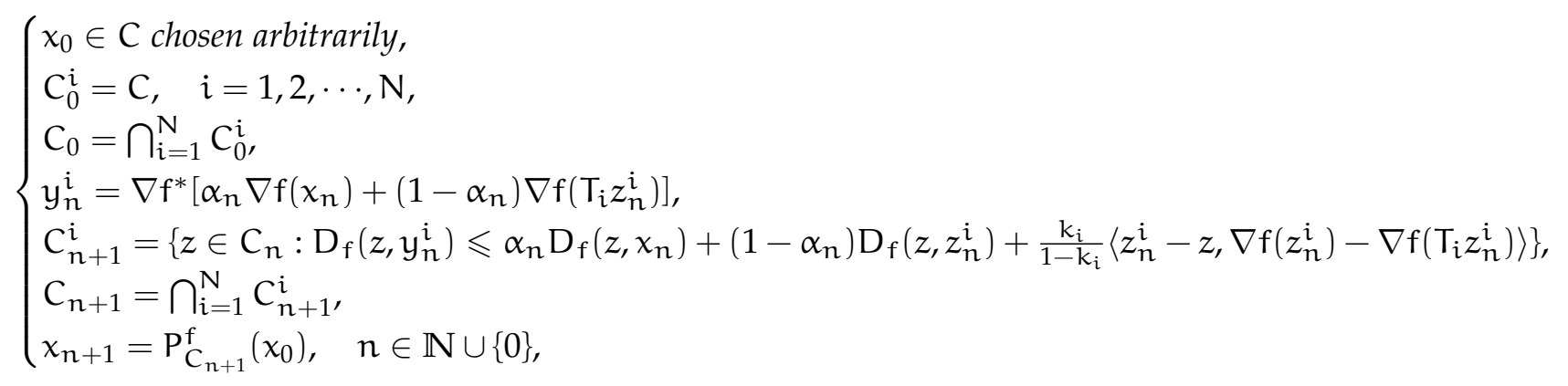

where $z_{n}^{i}=x_{n}+e_{n}^{i}, k_{i} \in[0,1)$, the sequences of errors $\left\{e_{n}^{i}\right\} \subset E$ satisfy $\lim _{n \rightarrow \infty} e_{n}^{i}=0$ for each $i=1,2, \cdots, N$, and $\left\{\alpha_{n}\right\} \subset[0,1]$ satisfies $\lim _{\inf _{n \rightarrow \infty}}\left(1-\alpha_{n}\right)>0$. Then the sequence $\left\{x_{n}\right\}$ converges strongly to $\widehat{p}=P_{F}^{f}\left(x_{0}\right)$, where $\mathrm{P}_{\mathrm{F}}^{\mathrm{f}}$ is the Bregman projection of $\mathrm{E}$ onto $\mathrm{F}$.

Proof. From Lemma 2.10, one has $F\left(T_{i}\right)$ is closed and convex for any $1 \leqslant i \leqslant N$. Then $F=\bigcap_{i=1}^{N} F\left(T_{i}\right)$ is also closed and convex. Therefore $\mathrm{P}_{\mathrm{F}}^{\mathrm{f}}\left(\mathrm{x}_{0}\right)$ is well-defined for every $\mathrm{x}_{0} \in \mathrm{C}$. Note that $\mathrm{C}_{0}=\mathrm{C}$ is closed and convex. Let $C_{m}$ is closed and convex for some $m \in \mathbb{N}$. For $z \in C_{m}$, we see that

$$
\begin{aligned}
D_{f}\left(z, y_{m}^{i}\right) \leqslant & \alpha_{m} D_{f}\left(z, x_{m}\right)+\left(1-\alpha_{m}\right) D_{f}\left(z, x_{m}+e_{m}^{i}\right) \\
& +\frac{k_{i}}{1-k_{i}}\left\langle x_{m}+e_{m}^{i}-z, \nabla f\left(x_{m}+e_{m}^{i}\right)-\nabla f\left(T_{i}\left(x_{m}+e_{m}^{i}\right)\right)\right\rangle
\end{aligned}
$$

is equivalent to

$$
\begin{aligned}
& \left\langle z, \frac{k_{i}}{1-k_{i}}\left[\nabla f\left(x_{m}+e_{m}^{i}\right)-\nabla f\left(T_{i}\left(x_{m}+e_{m}^{i}\right)\right]+\alpha_{m} \nabla f\left(x_{m}\right)+\left(1-\alpha_{m}\right) \nabla f\left(x_{m}+e_{m}^{i}\right)-\nabla f\left(y_{m}^{i}\right)\right\rangle\right. \\
& \quad \leqslant f\left(y_{m}^{i}\right)-\alpha_{m} f\left(x_{m}\right)-\left(1-\alpha_{m}\right) f\left(x_{m}+e_{m}^{i}\right)-\left\langle y_{m}^{i}, \nabla f\left(y_{m}^{i}\right)\right\rangle+\alpha_{m}\left\langle x_{m}, \nabla f\left(x_{m}\right)\right\rangle \\
& \left.\quad+\left(1-\alpha_{m}\right)\left\langle x_{m}+e_{m}^{i}, \nabla f\left(x_{m}+e_{m}^{i}\right)\right\rangle+\frac{k_{i}}{1-k_{i}}\left\langle x_{m}+e_{m}^{i}, \nabla f\left(x_{m}+e_{m}^{i}\right)-\nabla f\left(T_{i}\left(x_{m}+e_{m}^{i}\right)\right)\right]\right\rangle .
\end{aligned}
$$

Hence, we see that $C_{m+1}$ is closed and convex. Therefore $C_{n}$ is closed and convex for all $n \in \mathbb{N} \cup\{0\}$.

We note that $F(T) \subset C=C_{0}$. Suppose that $F(T) \subset C_{m}$ for some $m \in \mathbb{N}$. From (2.2), for any $p \in F(T) \subset C_{m}$, we obtain

$$
\begin{aligned}
D_{f}\left(p, y_{m}^{i}\right)= & D_{f}\left(p, \nabla f^{*}\left[\alpha_{m} \nabla f\left(x_{m}\right)+\left(1-\alpha_{m}\right) \nabla f\left(T_{i}\left(x_{m}+e_{m}\right)\right)\right]\right) \\
= & V\left(p, \alpha_{m} \nabla f\left(x_{m}\right)+\left(1-\alpha_{m}\right) \nabla f\left(T_{i}\left(x_{m}+e_{m}\right)\right)\right) \\
= & f(p)-\left\langle p, \alpha_{m} \nabla f\left(x_{m}\right)+\left(1-\alpha_{m}\right) \nabla f\left(T_{i}\left(x_{m}+e_{m}\right)\right)\right\rangle \\
& +f^{*}\left(\alpha_{m} \nabla f\left(x_{m}\right)+\left(1-\alpha_{m}\right) \nabla f\left(T_{i}\left(x_{m}+e_{m}\right)\right)\right) \\
\leqslant & \alpha_{m}\left[f(p)-\left\langle p, \nabla f\left(x_{m}\right)\right\rangle+f^{*}\left(\nabla f\left(x_{m}\right)\right)\right] \\
& +\left(1-\alpha_{m}\right)\left[f(p)-\left\langle p, \nabla f\left(T_{i}\left(x_{m}+e_{m}\right)\right)\right\rangle+f^{*}\left(\nabla f\left(T_{i}\left(x_{m}+e_{m}\right)\right)\right)\right] \\
= & \alpha_{m} V\left(p, \nabla f\left(x_{m}\right)\right)+\left(1-\alpha_{m}\right) V\left(p, \nabla f\left(T_{i}\left(x_{m}+e_{m}\right)\right)\right) \\
= & \alpha_{m} D_{f}\left(p, x_{m}\right)+\left(1-\alpha_{m}\right) D_{f}\left(p, T_{i}\left(x_{m}+e_{m}\right)\right) \\
\leqslant & \alpha_{m} D_{f}\left(p, x_{m}\right)+\left(1-\alpha_{m}\right)\left[D_{f}\left(p, x_{m}+e_{m}\right)+k_{i} D_{f}\left(x_{m}+e_{m}, T_{i}\left(x_{m}+e_{m}\right)\right)\right] \\
\leqslant & \alpha_{m} D_{f}\left(p, x_{m}\right)+\left(1-\alpha_{m}\right) D_{f}\left(p, x_{m}+e_{m}\right) \\
& +\frac{\left(1-\alpha_{m}\right) k_{i}}{1-k_{i}}\left\langle x_{m}+e_{m}-p, \nabla f\left(x_{m}+e_{m}\right)-\nabla f\left(T_{i}\left(x_{m}+e_{m}\right)\right)\right\rangle, \\
\leqslant & \alpha_{m} D_{f}\left(p, x_{m}\right)+\left(1-\alpha_{m}\right) D_{f}\left(p, x_{m}+e_{m}\right) \\
& +\frac{k_{i}}{1-k_{i}}\left\langle x_{m}+e_{m}-p, \nabla f\left(x_{m}+e_{m}\right)-\nabla f\left(T_{i}\left(x_{m}+e_{m}\right)\right)\right\rangle .
\end{aligned}
$$

This implies that $p \in C_{m+1}$. Thus, we have $F \subset C_{n}$ for all $n \in \mathbb{N} \cup\{0\}$. 

has

Next, we show that $\lim _{n \rightarrow \infty} D_{f}\left(x_{n}, x_{0}\right)$ exists. In fact, since $x_{n}=P_{C_{n}}^{f}\left(x_{0}\right)$, from Lemma 2.1 (c), one

$$
D_{f}\left(x_{n}, x_{0}\right)=D_{f}\left(P_{C_{n}}^{f}\left(x_{0}\right), x_{0}\right) \leqslant D_{f}\left(p, x_{0}\right)-D_{f}\left(p, P_{C_{n}}^{f}\left(x_{0}\right)\right) \leqslant D_{f}\left(p, x_{0}\right),
$$

for each $p \in F(T)$ and for each $n \geqslant 1$. Therefore, $\left\{D_{f}\left(x_{n}, x_{0}\right)\right\}_{\mathfrak{n} \in \mathbb{N}}$ is bounded. In view of Lemma 2.3, one has $\left\{x_{n}\right\}$ is also bounded. On the other hand, noticing that $x_{n}=P_{C_{n}}^{f}\left(x_{0}\right)$ and $x_{n+1}=P_{C_{n+1}}^{f}\left(x_{0}\right) \in C_{n+1} \subset$ $C_{n}$, one has $D_{f}\left(x_{n}, x_{0}\right) \leqslant D_{f}\left(x_{n+1}, x_{0}\right)$ for all $n \geqslant 1$. This implies that $\left\{D_{f}\left(x_{n}, x_{0}\right)\right\}_{n \in \mathbb{N}}$ is a nondecreasing sequence. Therefore, $\lim _{n \rightarrow \infty} D_{f}\left(x_{n}, x_{0}\right)$ exists. Since $\left\{x_{n}\right\}$ is bounded and $E$ is reflexive, there exists a subsequence $\left\{x_{n_{i}}\right\} \subset\left\{x_{n}\right\}$ such that $x_{n_{i}} \rightarrow \widehat{p} \in C=C_{0}$. Since $C_{n}$ is closed and convex and $C_{n+1} \subset C_{n}$, this implies that $C_{n}$ is weakly closed and $\widehat{p} \in C_{n}$ for all $n \geqslant 0$. In view of $x_{n_{i}}=P_{C_{n_{i}}}^{f}\left(x_{0}\right)$, one has

$$
D_{f}\left(x_{n_{i}}, x_{0}\right) \leqslant D_{f}\left(\widehat{p}, x_{0}\right), \quad \forall n_{i} \geqslant 1 \text {. }
$$

Since $f$ is a lower semi-continuous function on convex set $C$, it is weakly lower semi-continuous on C. Hence we have

$$
\begin{aligned}
\liminf _{i \rightarrow \infty} D_{f}\left(x_{n_{i}}, x_{0}\right) & =\liminf _{i \rightarrow \infty}\left\{f\left(x_{n_{i}}\right)-f\left(x_{0}\right)-\left\langle\nabla f\left(x_{0}\right), x_{n_{i}}-x_{0}\right\rangle\right\} \\
& \geqslant f(\widehat{p})-f\left(x_{0}\right)-\left\langle\nabla f\left(x_{0}\right), \widehat{p}-x_{0}\right\rangle \\
& =D_{f}\left(\widehat{p}, x_{0}\right) .
\end{aligned}
$$

Therefore, one has

$$
D_{f}\left(\widehat{p}, x_{0}\right) \leqslant \liminf _{i \rightarrow \infty} D_{f}\left(x_{n_{i}}, x_{0}\right) \leqslant \limsup _{i \rightarrow \infty} D_{f}\left(x_{n_{i}}, x_{0}\right) \leqslant D_{f}\left(\widehat{p}, x_{0}\right),
$$

which implies that

$$
\lim _{i \rightarrow \infty} D_{f}\left(x_{n_{i}}, x_{0}\right)=D_{f}\left(\widehat{p}, x_{0}\right) .
$$

In view of Lemma 2.1 (c), we have that

$$
D_{f}\left(\widehat{p}, x_{n_{i}}\right) \leqslant D_{f}\left(\widehat{p}, x_{0}\right)-D_{f}\left(x_{n_{i}}, x_{0}\right),
$$

by taking $i \rightarrow \infty$ in the above inequality and (3.1), we obtain that

$$
\lim _{i \rightarrow \infty} D_{f}\left(\widehat{p}, x_{n_{i}}\right)=0,
$$

which implies from Lemma 2.2 and (2.3) that

$$
\lim _{i \rightarrow \infty} x_{n_{i}}=\widehat{p} .
$$

On the other hand, notice that $\left\{D_{f}\left(x_{n}, x_{0}\right)\right\}$ is convergent. This together with (3.1) implies that

$$
\lim _{n \rightarrow \infty} D_{f}\left(x_{n}, x_{0}\right)=D_{f}\left(\widehat{p}, x_{0}\right) .
$$

From Lemma 2.1 (c), we also have

$$
D_{f}\left(\widehat{p}, x_{n}\right) \leqslant D_{f}\left(\widehat{p}, x_{0}\right)-D_{f}\left(x_{n}, x_{0}\right) .
$$

By taking $n \rightarrow \infty$ in the above inequality and (3.2), we obtain

$$
\lim _{n \rightarrow \infty} D_{f}\left(\widehat{p}, x_{n}\right)=0,
$$

which implies from Lemma 2.2 and (2.3) that

$$
\lim _{n \rightarrow \infty} x_{n}=\widehat{p} .
$$


Since $e_{n}^{i} \rightarrow 0$ as $n \rightarrow \infty$ for any $1 \leqslant i \leqslant N$, it is obvious from (3.3) that

$$
\lim _{n \rightarrow \infty}\left(x_{n}+e_{n}^{i}\right)=\widehat{p}, \quad \forall 1 \leqslant i \leqslant N .
$$

Now, we are in a position to show that the limit of $\left\{x_{n}\right\}_{n \in \mathbb{N}}$ belongs to $F=\bigcap_{i=1}^{N} F\left(T_{i}\right)$. Since $x_{n}=$ $P_{C_{n}}^{f} x_{0}$, one has from Lemma 2.1 (c) that

$$
D_{f}\left(x_{n+1}, x_{n}\right) \leqslant D_{f}\left(x_{n+1}, x_{0}\right)-D_{f}\left(x_{n}, x_{0}\right) .
$$

Hence, we have

$$
\lim _{n \rightarrow \infty} D_{f}\left(x_{n+1}, x_{n}\right)=0 .
$$

Since $f$ is totally convex on bounded subsets of $E, f$ is sequentially consistent. It follows from (3.4) that

$$
\lim _{n \rightarrow \infty}\left\|x_{n+1}-x_{n}\right\|=0 .
$$

For any $i=1,2, \cdots, N$, it follows from the definition of the Bregman distance that

$$
\begin{aligned}
D_{f}\left(x_{n}, x_{n}+e_{n}^{i}\right) & =f\left(x_{n}\right)-f\left(x_{n}+e_{n}^{i}\right)-\left\langle x_{n}-\left(x_{n}+e_{n}^{i}\right), \nabla f\left(x_{n}+e_{n}^{i}\right)\right\rangle \\
& =f\left(x_{n}\right)-f\left(x_{n}+e_{n}^{i}\right)+\left\langle e_{n}^{i}, \nabla f\left(x_{n}+e_{n}^{i}\right)\right\rangle .
\end{aligned}
$$

The function $f$ is bounded on bounded subsets of $E$ and therefore $\nabla f$ is also bounded subset of $E$. In addition, $f$ is uniformly Fréchet differentiable and therefore $f$ is uniformly continuous on bounded subsets. Hence, from $\lim _{n \rightarrow \infty} e_{n}^{i}=0$, one has that

$$
\lim _{n \rightarrow \infty} D_{f}\left(x_{n}, x_{n}+e_{n}^{i}\right)=0 .
$$

For any $i=1,2, \cdots, N$, it follows from the three point identity that

$$
D_{f}\left(x_{n+1}, x_{n}+e_{n}^{i}\right)=D_{f}\left(x_{n+1}, x_{n}\right)+D_{f}\left(x_{n}, x_{n}+e_{n}^{i}\right)+\left\langle x_{n+1}-x_{n}, \nabla f\left(x_{n}\right)-\nabla f\left(x_{n}+e_{n}^{i}\right)\right\rangle .
$$

Since $\nabla f$ is bounded on bounded subsets of $E$, it implies from (3.4), (3.5), and (3.6) that

$$
\lim _{n \rightarrow \infty} D_{f}\left(x_{n+1}, x_{n}+e_{n}^{i}\right)=0 .
$$

On the other hand, from the fact $x_{n+1} \in C_{n+1}$, it follows

$$
D_{f}\left(x_{n+1}, y_{n}^{i}\right) \leqslant \alpha_{n} D_{f}\left(x_{n+1}, x_{n}\right)+\left(1-\alpha_{n}\right) D_{f}\left(x_{n+1}, z_{n}^{i}\right)+\frac{k_{i}}{1-k_{i}}\left\langle z_{n}^{i}-x_{n+1}, \nabla f\left(z_{n}^{i}\right)-\nabla f\left(T_{i}\left(z_{n}^{i}\right)\right)\right\rangle,
$$

where $z_{n}^{i}=x_{n}+e_{n}^{i}$. It implies from (3.4), (3.5), (3.7), and $\lim _{n \rightarrow \infty} e_{n}^{i}=0$ that

$$
\lim _{n \rightarrow \infty} D_{f}\left(x_{n+1}, y_{n}^{i}\right)=0, \quad \forall i=1,2, \cdots, N .
$$

Since $f$ is totally convex on bounded subsets of $E, f$ is sequentially consistent. It follows from (3.8) that

$$
\lim _{n \rightarrow \infty}\left\|x_{n+1}-y_{n}^{i}\right\|=0, \quad \forall i=1,2, \cdots, N,
$$

which implies from (3.5) that

$$
\lim _{n \rightarrow \infty}\left\|x_{n}-y_{n}^{i}\right\|=0, \quad \forall i=1,2, \cdots, N .
$$

From the uniform continuity of $\nabla f$, one has

$$
\lim _{n \rightarrow \infty}\left\|\nabla f\left(x_{n}\right)-\nabla f\left(y_{n}^{i}\right)\right\|=0, \quad \forall i=1,2, \cdots, N .
$$


Since

$$
y_{n}^{i}=\nabla f^{*}\left[\alpha_{n} \nabla f\left(x_{n}\right)+\left(1-\alpha_{n}\right) \nabla f\left(T_{i}\left(x_{n}+e_{n}^{i}\right)\right)\right] .
$$

One sees from (3.9) and $\liminf _{n \rightarrow \infty}\left(1-\alpha_{n}\right)>0$ that

$$
\left.\lim _{n \rightarrow \infty}\left\|\nabla f\left(x_{n}\right)-\nabla f\left(T_{i}\left(x_{n}+e_{n}^{i}\right)\right)\right\|=\lim _{n \rightarrow \infty} \frac{1}{1-\alpha_{n}} \| \nabla f\left(x_{n}\right)-\nabla f\left(y_{n}^{i}\right)\right) \|=0
$$

Since $f$ is strongly coercive and uniformly convex on bounded subsets of $E, f^{*}$ is uniformly Fréchet differentiable on bounded sets. Moreover, $f^{*}$ is bounded on bounded sets, and from (3.10) one has

$$
\lim _{n \rightarrow \infty}\left\|x_{n}-T_{i}\left(x_{n}+e_{n}^{i}\right)\right\|=0,
$$

which implies from $\lim _{n \rightarrow \infty} e_{n}^{i}=0$ that

$$
\lim _{n \rightarrow \infty}\left\|\left(x_{n}+e_{n}^{i}\right)-T_{i}\left(x_{n}+e_{n}^{i}\right)\right\|=0, \quad \forall i=1,2, \cdots, N .
$$

Since $T_{i}$, for $i=1,2, \cdots, N$, is closed, and $\lim _{n \rightarrow \infty} x_{n}=\lim _{n \rightarrow \infty}\left(x_{n}+e_{n}^{i}\right)=\widehat{p}$, one obtains that $\widehat{p} \in$ $\bigcap_{i=1}^{N} F\left(T_{i}\right)=F$.

Finally, we prove $\widehat{p}=P_{F(T)}^{f}\left(x_{0}\right)$. From $x_{n}=P_{C_{n}}^{f} x_{0}$, one has

$$
\left\langle y-x_{n}, \nabla f\left(x_{0}\right)-\nabla f\left(x_{n}\right)\right\rangle \leqslant 0, \quad \forall y \in C_{n} .
$$

Since $F \subset C_{n}$ for each $n \in \mathbb{N}$, one obtains

$$
\left\langle y-x_{n}, \nabla f\left(x_{0}\right)-\nabla f\left(x_{n}\right)\right\rangle \leqslant 0, \quad \forall y \in F .
$$

Taking $n \rightarrow \infty$ in (3.11), one has

$$
\left\langle y-\widehat{p}, \nabla f\left(x_{0}\right)-\nabla f(\widehat{p})\right\rangle \leqslant 0, \quad \forall y \in F .
$$

In view of Lemma 2.1 (a) and Lemma $2.4(b)$, one has $\widehat{p}=\mathrm{P}_{\mathrm{F}(\mathrm{T})}^{\mathrm{f}}\left(\mathrm{x}_{0}\right)$. This completes the proof of Theorem 3.1 .

For single closed and Bregman quasi-strict pseudo-contraction $T$, we have the following result.

Corollary 3.2. Let $\mathrm{E}$ be a real reflexive Banach space, and $\mathrm{C}$ be a nonempty, closed, and convex subset of $\mathrm{E}$. Let $\mathrm{f}: \mathrm{E} \rightarrow \mathbb{R}$ be a strongly coercive Legendre function which is bounded, uniformly Fréchet differentiable, and totally convex on bounded subsets of $\mathrm{E}$, and $\mathrm{T}: \mathrm{C} \rightarrow \mathrm{C}$ be a Bregman quasi-k-strict pseudo-contraction such that $\mathrm{F}(\mathrm{T}) \neq \emptyset$. Let $\left\{x_{n}\right\}$ be a sequence generated by the following iterative algorithm:

$$
\left\{\begin{array}{l}
x_{0} \in C \text { chosen arbitrarily, } \\
C_{0}=C, \\
y_{n}=\nabla f^{*}\left[\alpha_{n} \nabla f\left(x_{n}\right)+\left(1-\alpha_{n}\right) \nabla f\left(T\left(z_{n}\right)\right],\right. \\
C_{n+1}=\left\{z \in C_{n}: D_{f}\left(z, y_{n}\right) \leqslant \alpha_{n} D_{f}\left(z, x_{n}\right)+\left(1-\alpha_{n}\right) D_{f}\left(z, z_{n}\right)+\frac{k}{1-k}\left\langle z_{n}-z, \nabla f\left(z_{n}\right)-\nabla f\left(T z_{n}\right)\right\rangle\right\}, \\
x_{n+1}=P_{C_{n+1}}^{f}\left(x_{0}\right), \quad n \in \mathbb{N} \cup\{0\},
\end{array}\right.
$$

where $z_{n}=x_{n}+e_{n}, k \in[0,1)$, the sequences of errors $\left\{e_{n}^{i}\right\} \subset E$ satisfy $\lim _{n \rightarrow \infty} e_{n}^{i}=0$ for each $i=1,2, \cdots, N$, and $\left\{\alpha_{n}\right\} \subset[0,1]$ satisfies $\liminf _{n \rightarrow \infty}\left(1-\alpha_{n}\right)>0$. Then the sequence $\left\{x_{n}\right\}$ converges strongly to $\widehat{p}=\mathrm{P}_{\mathrm{F}(\mathrm{T})}^{f}\left(\mathrm{x}_{0}\right)$, where $\mathrm{P}_{\mathrm{F}(\mathrm{T})}^{\mathrm{f}}$ is the Bregman projection of $\mathrm{E}$ onto $\mathrm{F}(\mathrm{T})$.

Setting $f(x)=\|x\|^{2}$ for all $x \in E$, then $\nabla f(x)=2 J x$ for all $x \in E$. Hence $D_{f}(x, y)$ reduces to the Lyapunov function $\phi(x, y)=\|x\|^{2}-2\langle x, J y\rangle+\|y\|^{2}$ for all $x, y \in E$, the Bregman projection $P_{C}^{f}(x)$ reduces to the generalized projection $\Pi_{C}$ from $E$ onto $C$ and the Bregman quasi-strict pseudo-contraction reduces to the strict quasi- $\phi$-pseudocontraction. So, by utilizing Theorem 3.1, the following result is obtained. 
Corollary 3.3. Let $\mathrm{E}$ be a real reflexive, uniformly smooth, and uniformly convex Banach space, and $\mathrm{C}$ be a nonempty, closed, and convex subset of $\mathrm{E}$. Suppose that $\mathrm{T}_{i}: \mathrm{C} \rightarrow \mathrm{C}$, where $\mathrm{i}=1,2, \ldots, \mathrm{N}$, is a finite family of closed strict quasi- $\phi-k_{i}-$ pseudocontraction such that $\mathrm{F}=\bigcap_{i=1}^{N} \mathrm{~F}\left(\mathrm{~T}_{i}\right) \neq \emptyset$. Let $\left\{\mathrm{x}_{n}\right\}$ be a sequence generated by the following iterative algorithm:

$$
\left\{\begin{array}{l}
x_{0} \in C \text { chosen arbitrarily, } \\
C_{0}^{i}=C, \quad i=1,2, \cdots, N, \\
C_{0}=\bigcap_{i=1}^{N} C_{0}^{i}, \\
y_{n}^{i}=J^{-1}\left[\alpha_{n} J x_{n}+\left(1-\alpha_{n}\right) J T_{i} z_{n}^{i}\right], \\
C_{n+1}^{i}=\left\{z \in C_{n}: \phi\left(z, y_{n}^{i}\right) \leqslant \alpha_{n} \phi\left(z, x_{n}\right)+\left(1-\alpha_{\mathfrak{n}}\right) \phi\left(z, z_{\mathfrak{n}}^{i}\right)+\frac{k_{i}}{1-k_{i}}\left\langle z_{n}^{i}-z, J z_{n}^{i}-J T_{i} z_{n}^{i}\right\rangle\right\}, \\
C_{n+1}=\bigcap_{i=1}^{N} C_{n+1}^{i}, \\
x_{n+1}=P_{C_{n+1}}^{f}\left(x_{0}\right), \quad n \in \mathbb{N} \cup\{0\},
\end{array}\right.
$$

where $z_{n}^{i}=x_{n}+e_{n}^{i}, k_{i} \in[0,1)$, the sequences of errors $\left\{e_{n}^{i}\right\} \subset E$ satisfy $\lim _{n \rightarrow \infty} e_{n}^{i}=0$ for each $i=1,2, \cdots, N$, and $\left\{\alpha_{n}\right\} \subset[0,1]$ satisfies $\liminf _{n \rightarrow \infty}\left(1-\alpha_{n}\right)>0$. Then the sequence $\left\{x_{n}\right\}$ converges strongly to $\widehat{p}=P_{F}^{f}\left(x_{0}\right)$, where $\mathrm{P}_{\mathrm{F}}^{\mathrm{f}}$ is the Bregman projection of $\mathrm{E}$ onto $\mathrm{F}$.

\section{Applications}

In this section, we give some applications of our main results.

\subsection{Solving convex feasibility problems}

First, we give an application to convex feasibility problems. It is clear that $F\left(\mathrm{P}_{\mathrm{K}_{i}}^{\mathrm{f}}\right)=\mathrm{K}_{i}$ for any $i=1,2,3, \cdots, N$. If the Legendre function $f$ is uniformly Fréchet differentiable and bounded on bounded subsets of $E$, then the Bregman projection $P_{K_{i}}^{f}$ is a closed Bregman relatively nonexpansive mapping, so is a closed Bregman quasi-strict pseudo-contraction.

Theorem 4.1. Let $\mathrm{E}$ be a real reflexive Banach space, and $\mathrm{C}$ be a nonempty, closed, and convex subset of $\mathrm{E}$. Let $\mathrm{f}: \mathrm{E} \rightarrow \mathbb{R}$ be a strongly coercive Legendre function which is bounded, uniformly Fréchet differentiable, and totally convex on bounded subsets of $\mathrm{E}$, and let $\mathrm{K}_{i}, i=1,2, \ldots, \mathrm{N}$, be a finite family of closed and nonempty subsets of $\mathrm{C}$ such that $\mathrm{F}=\bigcap_{i=1}^{\mathrm{N}} \mathrm{K}_{i} \neq \emptyset$. Let $\left\{\mathrm{x}_{\mathrm{n}}\right\}$ be a sequence generated by the following iterative algorithm:

$$
\left\{\begin{array}{l}
x_{0} \in C \text { chosen arbitrarily, } \\
C_{0}^{i}=C, \quad i=1,2, \cdots, N, \\
C_{0}=\bigcap_{i=1}^{N} C_{0}^{i} \\
y_{n}^{i}=\nabla f^{*}\left[\alpha_{n} \nabla f\left(x_{n}\right)+\left(1-\alpha_{n}\right) \nabla f\left(P_{K_{i}}\left(x_{n}+e_{n}^{i}\right)\right)\right], \\
\left.C_{n+1}^{i}=\left\{z \in C_{n}: D_{f}\left(z, y_{n}^{i}\right) \leqslant \alpha_{n} D_{f}\left(z, x_{n}\right)+\left(1-\alpha_{n}\right) D_{f}\left(z, x_{n}+e_{n}^{i}\right)\right\rangle\right\}, \\
C_{n+1}=\bigcap_{i=1}^{N} C_{n+1}^{i}, \\
x_{n+1}=P_{C_{n+1}}^{f}\left(x_{0}\right), \quad n \in \mathbb{N} \cup\{0\},
\end{array}\right.
$$

where the sequences of errors $\left\{e_{n}^{i}\right\} \subset E$ satisfy $\lim _{n \rightarrow \infty} e_{n}^{i}=0$ for each $i=1,2, \cdots, N$, and $\left\{\alpha_{n}\right\} \subset[0,1]$ satisfies $\liminf _{n \rightarrow \infty}\left(1-\alpha_{n}\right)>0$. Then the sequence $\left\{x_{n}\right\}$ converges strongly to $\widehat{p}=P_{F}^{f}\left(x_{0}\right)$, where $P_{F}^{f}$ is the Bregman projection of $\mathrm{E}$ onto $\mathrm{F}$.

\subsection{Solving zeroes of maximal monotone operators}

Let $A$ be a mapping of $E$ into $2^{E^{*}}$. The effective domain of $A$ is denoted by $\operatorname{dom}(A)$, that is, $\operatorname{dom}(A)=$ $\{x \in E: A x \neq \emptyset\}$. The range of $A$ is denoted by $\operatorname{ran}(A)$, that is, $\operatorname{ran}(A)=\{A x: x \in \operatorname{dom}(A)\}$. A mapping $A: E \rightarrow 2^{E^{*}}$ is said to be monotone if for any $x, y \in \operatorname{dom}(A)$, we have

$$
u \in A x, v \in A y \Rightarrow\langle u-v, x-y\rangle \geqslant 0 .
$$


A monotone mapping $A$ is said to be maximal if graph $A$, the graph of $A$, is not a proper subset of the graph of any other monotone mapping.

Let $E$ be a real reflexive Banach space, and $A: E \rightarrow 2^{E^{*}}$ be a maximal monotone operator. The problem of finding an element $x \in E$ such that $0^{*} \in A x$ is very important in optimization theory and related fields. Recall that the resolvent of $A$, denoted by $\operatorname{Res}_{A}^{f}: E \rightarrow 2^{E}$, is defined as follows:

$$
\operatorname{Res}_{A}^{f}(x)=(\nabla f+A)^{-1} \circ \nabla f(x) .
$$

It is well-known that the fixed point set of the resolvent $\operatorname{Res}_{A}^{f}$ is equal to the set of zeroes of the mapping $A$, that is, $F\left(\operatorname{Res}_{A}^{f}\right)=A^{-1}\left(0^{*}\right)$. In fact,

$$
\begin{aligned}
u \in F\left(\operatorname{Res}_{A}^{f}\right) \Leftrightarrow u=\operatorname{Res}_{A}^{f}(u)=(\nabla f+A)^{-1} \circ \nabla f(u) & \Leftrightarrow \nabla f(u) \in \nabla f(u)+A(u) \\
& \Leftrightarrow 0^{*} \in A(u) \Leftrightarrow u \in(A)^{-1} 0^{*} .
\end{aligned}
$$

Since $\operatorname{Res}_{A}^{f}$ is a closed Bregman quasi-strict pseudo-contraction, we find the following result immediately.

Theorem 4.2. Let $\mathrm{E}$ be a real reflexive Banach space with the dual $\mathrm{E}^{*}, \mathrm{~A}_{i}: \mathrm{E} \rightarrow 2^{\mathrm{E}^{*}}, i=1,2, \cdots, \mathrm{N}$, be a finite family of maximal monotone operators with $\mathrm{F}=\bigcap_{i=1}^{N} A_{i}^{-1}\left(0^{*}\right) \neq \emptyset$. Let $\mathrm{f}: \mathrm{E} \rightarrow \mathbb{R}$ be a Legendre function which is bounded, uniformly Fréchet differentiable, and totally convex on bounded subsets of $\mathrm{E}$. Let $\operatorname{Res}_{A_{i}}^{\mathrm{f}}: \mathrm{E} \rightarrow 2^{\mathrm{E}}$ be the resolvent with respect to $A_{i}$. Let $\left\{x_{n}\right\}$ be a sequence generated by the following iterative algorithm:

$$
\left\{\begin{array}{l}
x_{0} \in C \text { chosen arbitrarily, } \\
C_{0}^{i}=C, \quad i=1,2, \cdots, N, \\
C_{0}=\bigcap_{i=1}^{N} C_{0}^{i}, \\
y_{n}^{i}=\nabla f^{*}\left[\alpha_{n} \nabla f\left(x_{n}\right)+\left(1-\alpha_{n}\right) \nabla f\left(\operatorname{Res}_{A_{i}}^{f}\left(x_{n}+e_{n}^{i}\right)\right)\right], \\
\left.C_{n+1}^{i}=\left\{z \in C_{n}: D_{f}\left(z, y_{n}^{i}\right) \leqslant \alpha_{n} D_{f}\left(z, x_{n}\right)+\left(1-\alpha_{n}\right) D_{f}\left(z, x_{n}+e_{n}^{i}\right)\right\rangle\right\}, \\
C_{n+1}=\bigcap_{i=1}^{N} C_{n+1}^{i}, \\
x_{n+1}=P_{C_{n+1}}^{f}\left(x_{0}\right), \quad n \in \mathbb{N} \cup\{0\},
\end{array}\right.
$$

where the sequences of errors $\left\{e_{n}^{i}\right\} \subset E$ satisfy $\lim _{n \rightarrow \infty} e_{n}^{i}=0$ for each $i=1,2, \cdots, N$, and $\left\{\alpha_{n}\right\} \subset[0,1]$ satisfies $\liminf _{n \rightarrow \infty}\left(1-\alpha_{n}\right)>0$. Then the sequence $\left\{x_{n}\right\}$ converges strongly to $\widehat{p}=\mathrm{P}_{\mathrm{F}}^{\mathrm{f}}\left(\mathrm{x}_{0}\right)$, where $\mathrm{P}_{\mathrm{F}}^{\mathrm{f}}$ is the Bregman projection of $\mathrm{E}$ onto $\mathrm{F}$.

\subsection{Solving minimizers of proper, lower semicontinuous, and convex functionals}

For a proper lower semicontinuous convex function $g: E \rightarrow(-\infty,+\infty]$, the subdifferential mapping $\partial g \subset E \times E^{*}$ of $g$ is defined as follows:

$$
\partial g=\left\{x^{*} \in E^{*}: g(y) \geqslant g(x)+\left\langle y-x, x^{*}\right\rangle, \forall y \in E\right\}, \quad \forall x \in E .
$$

From Rockafellar [19], we know that $\partial g$ is maximal monotone. It is easy to verify that $0^{*} \in \partial g(v)$ if and only if $g(v)=\min _{x \in E} g(x)$. Emulating (4.1) the resolvent of $\partial g$, denoted by $\operatorname{Res}_{\partial g}^{f}: E \rightarrow 2^{E}$, is defined as follows:

$$
\operatorname{Res}_{\partial g}^{f}(x)=(\nabla f+\partial g)^{-1} \circ \nabla f(x) .
$$

Theorem 4.3. Let $\mathrm{E}$ be a real reflexive Banach space with the dual $\mathrm{E}^{*}$, and $\mathrm{f}: \mathrm{E} \rightarrow \mathbb{R}$ be a Legendre function which is bounded, uniformly Fréchet differentiable, and totally convex on bounded subsets of $\mathrm{E}$. Let $\mathrm{g}_{\mathrm{i}}: \mathrm{E} \rightarrow(-\infty, \infty]$, $i=1,2, \cdots, N$, be a finite family of proper, lower semicontinuous, and convex functions, $\partial g_{i}$ the subdifferential mapping of $g_{i}$, and $\operatorname{Res}_{\partial g_{i}}^{f}$ the resolvent of $\partial g_{i}$. Assume that $F=\bigcap_{i=1}^{N}\left(\partial g_{i}\right)^{-1}\left(0^{*}\right) \neq \emptyset$. Let $\left\{x_{n}\right\}$ be a sequence generated by the following iterative algorithm: 


$$
\left\{\begin{array}{l}
x_{0} \in C \text { chosen arbitrarily, } \\
C_{0}^{i}=C, \quad i=1,2, \cdots, N, \\
C_{0}=\bigcap_{i=1}^{N} C_{0}^{i}, \\
y_{n}^{i}=\nabla f^{*}\left[\alpha_{n} \nabla f\left(x_{n}\right)+\left(1-\alpha_{n}\right) \nabla f\left(\operatorname{Res}_{\partial g_{i}}^{f}\left(x_{n}+e_{n}^{i}\right)\right)\right], \\
\left.C_{n+1}^{i}=\left\{z \in C_{n}: D_{f}\left(z, y_{n}^{i}\right) \leqslant \alpha_{n} D_{f}\left(z, x_{n}\right)+\left(1-\alpha_{n}\right) D_{f}\left(z, x_{n}+e_{n}^{i}\right)\right\rangle\right\}, \\
C_{n+1}=\bigcap_{i=1}^{N} C_{n+1}^{i}, \\
x_{n+1}=P_{C_{n+1}}^{f}\left(x_{0}\right), \quad n \in \mathbb{N} \cup\{0\},
\end{array}\right.
$$

where the sequences of errors $\left\{e_{n}^{i}\right\} \subset E$ satisfy $\lim _{n \rightarrow \infty} e_{n}^{i}=0$ for each $i=1,2, \cdots, N$, and $\left\{\alpha_{n}\right\} \subset[0,1]$ satisfies $\liminf _{n \rightarrow \infty}\left(1-\alpha_{n}\right)>0$. Then the sequence $\left\{x_{n}\right\}$ converges strongly to $\widehat{p}=P_{F}^{f}\left(x_{0}\right)$, where $P_{F}^{f}$ is the Bregman projection of $\mathrm{E}$ onto $\mathrm{F}$.

\subsection{Solving equilibrium problems}

Let $C$ be a nonempty, closed, and convex subset of a real reflexive Banach space $E$. Let $G: C \times C \rightarrow \mathbb{R}$ be a bifunction that satisfies the following conditions:

(A1) $\mathrm{G}(x, x)=0$ for all $x \in C$;

(A2) $G$ is monotone, i.e., $G(x, y)+G(y, x) \leqslant 0$ for all $x, y \in C$;

(A3) for all $x, y, z \in C$, limsup $\operatorname{sul}_{t \downarrow} G(t z+(1-t) x, y) \leqslant G(x, y)$;

(A4) for each $x \in C, G(x, \cdot)$ is convex and lower semicontinuous.

The "so-called" equilibrium problem corresponding to $G$ is to find $\bar{x} \in \mathrm{C}$ such that $\mathrm{G}(\bar{x}, y) \geqslant 0, \forall y \in C$. The set of its solutions is denoted by $\operatorname{EP}(\mathrm{G})$. The resolvent of a bifunction $\mathrm{G}: \mathrm{C} \times \mathrm{C} \rightarrow \mathbb{R}$ is the operator $\operatorname{Res}_{\mathrm{G}}^{\mathrm{f}}: \mathrm{E} \rightarrow 2^{\mathrm{C}}$ defined by

$$
\operatorname{Res}_{\mathrm{G}}^{\mathrm{f}}(\mathrm{x})=\{z \in \mathrm{C}: \mathrm{G}(z, \mathrm{y})+\langle\nabla f(z)-\nabla f(x), y-z\rangle \geqslant 0, \quad \forall \mathrm{y} \in \mathrm{C}\} .
$$

It is well-known that $\operatorname{Res}_{G}^{f}$ has the following properties:

(1) $\operatorname{Res}_{\mathrm{G}}^{f}$ is single-valued;

(2) the set of fixed points of $\operatorname{Res}_{G}^{f}$ is the solution set of the corresponding equilibrium problem, i.e., $F\left(\operatorname{Res}_{\mathrm{G}}^{\mathrm{f}}\right)=\mathrm{EP}(\mathrm{G})$

(3) $\operatorname{Res}_{G}^{f}$ is a closed Bregman quasi-nonexpansive mapping, so is a closed Bregman quasi-strict pseudocontraction.

Theorem 4.4. Let $\mathrm{E}$ be a real reflexive Banach space, and $\mathrm{C}$ be a nonempty, closed, and convex subset of $\mathrm{E}$. Let $\mathrm{G}_{i}: \mathrm{C} \times \mathrm{C} \rightarrow \mathbb{R}, i=1,2, \cdots, \mathrm{N}$, be a finite family of bifunctions that satisfy conditions (A1)-(A4) such that $\mathrm{F}=\bigcap_{i=1}^{\mathrm{N}} \mathrm{EP}\left(\mathrm{G}_{i}\right) \neq \emptyset$. Let $\mathrm{f}: \mathrm{E} \rightarrow \mathbb{R}$ be a Legendre function which is bounded, uniformly Fréchet differentiable, and totally convex on bounded subsets of $\mathrm{E}$, and $\operatorname{Res}_{\mathrm{G}_{i}}^{\mathrm{f}}: \mathrm{E} \rightarrow 2^{\mathrm{C}}$ be resolvent operator. Let $\left\{\mathrm{x}_{\mathrm{n}}\right\}$ be a sequence generated by the following iterative algorithm:

$$
\left\{\begin{array}{l}
x_{0} \in C \text { chosen arbitrarily, } \\
C_{0}^{i}=C, \quad i=1,2, \ldots, N, \\
C_{0}=\bigcap_{i=1}^{N} C_{0}^{i}, \\
y_{n}^{i}=\nabla f^{*}\left[\alpha_{n} \nabla f\left(x_{n}\right)+\left(1-\alpha_{n}\right) \nabla f\left(\operatorname{Res}_{G_{i}}^{f}\left(x_{n}+e_{n}^{i}\right)\right],\right. \\
\left.C_{n+1}^{i}=\left\{z \in C_{n}: D_{f}\left(z, y_{n}^{i}\right) \leqslant \alpha_{n} D_{f}\left(z, x_{n}\right)+\left(1-\alpha_{n}\right) D_{f}\left(z, x_{n}+e_{n}^{i}\right)\right\rangle\right\}, \\
C_{n+1}=\bigcap_{i=1}^{N} C_{n+1}^{i}, \\
x_{n+1}=P_{C_{n+1}}^{f}\left(x_{0}\right), \quad n \in \mathbb{N} \cup\{0\},
\end{array}\right.
$$

where the sequences of errors $\left\{e_{n}^{i}\right\} \subset E$ satisfy $\lim _{n \rightarrow \infty} e_{n}^{i}=0$ for each $i=1,2, \cdots, N$, and $\left\{\alpha_{n}\right\} \subset[0,1]$ satisfies $\liminf _{n \rightarrow \infty}\left(1-\alpha_{n}\right)>0$. Then the sequence $\left\{x_{n}\right\}$ converges strongly to $\widehat{p}=P_{F}^{f}\left(x_{0}\right)$, where $P_{F}^{f}$ is the Bregman projection of $\mathrm{E}$ onto $\mathrm{F}$. 
Proof. Since $\operatorname{Res}_{\mathrm{G}_{i}}^{f}$ is a closed Bregman quasi-strict pseudo-contraction for each $i=1,2, \cdots, N$, by applying Theorem 3.1, the sequence $\left\{x_{n}\right\}$ converges strongly to $\widehat{p}=\mathrm{P}_{\mathrm{F}}^{\mathrm{f}}\left(\mathrm{x}_{0}\right)$.

\section{Numerical examples}

In this section, in order to demonstrate the effectiveness, realization and convergence of the algorithm in Theorem 3.1, we consider the following example and give the visualization result by utilizing MATLAB 7.0 software.

Example 5.1. Let $E=\mathbb{R}, C=[0,1], f(x)=x^{2}, S x=\sin x$, and $T x=\sin \left(\frac{1}{2} x\right)$. Then both $S$ and $T$ are Bregman quasi-strict pseudo-contractions with the fixed point 0.

Proof. From the definition of $S$, one easily sees that $F(S)=\{0\}$. By the definition of the Bregman distance $D_{f}(\cdot, \cdot)$, we compute that

$$
\begin{aligned}
D_{f}(0, S x) & =f(0)-f(S x)-\langle 0-S x, \nabla f(S x)\rangle \\
& =0-\sin ^{2} x-\langle 0-\sin x, 2 \sin x\rangle \\
& =\sin ^{2} x \\
D_{f}(0, x) & =f(0)-f(x)-\langle 0-x, \nabla f(x)\rangle \\
& =0-x^{2}-\langle 0-x, 2 x\rangle \\
& =x^{2}
\end{aligned}
$$

and

$$
\begin{aligned}
D_{f}(x, S x) & =f(x)-f(S x)-\langle x-S x, \nabla f(S x)\rangle \\
& =x^{2}-\sin ^{2} x-\langle x-\sin x, 2 \sin x\rangle \\
& =x^{2}+\sin ^{2} x-2 x \sin x \geqslant 0 .
\end{aligned}
$$

From (5.1), (5.2), and (5.3), for any $k \in[0,1)$ one obtains that

$$
D_{f}(0, S x)=\sin ^{2} x \leqslant x^{2} \leqslant x^{2}+k\left(x^{2}+\sin ^{2} x-2 x \sin x\right)=D_{f}(0, x)+k D_{f}(x, S x) .
$$

From the definition of Bregman quasi-strict pseudo-contractions, hence $S$ is a Bregman quasi-strict pseudo-contraction. Similarly, one can obtain that T is also a Bregman quasi-strict pseudo-contraction.

In the algorithm of Theorem 3.1, set $e_{\mathfrak{n}}^{i} \equiv 0$ for $i=1,2$. By using Example 5.1, the algorithm of Theorem 3.1 can be simplified as

$$
\left\{\begin{array}{l}
x_{0} \in C \text { chosen arbitrarily, } \\
C_{0}^{i}=C=[0,1], \quad i=1,2, \\
C_{0}=C_{0}^{1} \bigcap C_{0}^{2}=[0,1] \\
y_{n}^{1}=\alpha_{n} x_{n}+\left(1-\alpha_{n}\right) \sin x_{n}, \\
y_{n}^{2}=\alpha_{n} x_{n}+\left(1-\alpha_{n}\right) \sin \frac{1}{2} x_{n}, \\
C_{n+1}^{1}=\left\{z \in C_{n}: z \leqslant \frac{\left(1-\kappa_{1}\right)\left(x_{n}^{2}-y_{n}^{2}\right)+\kappa_{1} x_{n}\left(x_{n}-\sin x_{n}\right)}{\kappa_{1}\left(x_{n}-\sin x_{n}\right)+2\left(1-\kappa_{1}\right)\left(x_{n}-y_{n}\right)}\right\} \\
C_{n+1}^{2}=\left\{z \in C_{n}: z \leqslant \frac{\left(1-\kappa_{2}\right)\left(x_{n}^{2}-y_{n}^{2}\right)+\kappa_{2} x_{n}\left(x_{n}-\sin \frac{1}{2} x_{n}\right)}{\kappa_{2}\left(x_{n}-\sin \frac{1}{2} x_{n}\right)+2\left(1-\kappa_{2}\right)\left(x_{n}-y_{n}\right)}\right\} \\
x_{n+1}=P_{C_{n+1}^{1} \cap C_{n+1}^{2}}^{f}\left(x_{0}\right), \quad n \in \mathbb{N} \cup\{0\},
\end{array}\right.
$$

In the following, for the same initial value $x_{0}=1$, the same parameter $\kappa_{i}=\frac{1}{2}, i=1,2$, and the different parametric sequence $\left\{\alpha_{n}\right\}$, we make simulations on the algorithm (5.4) by MATLAB 7.0 software. 


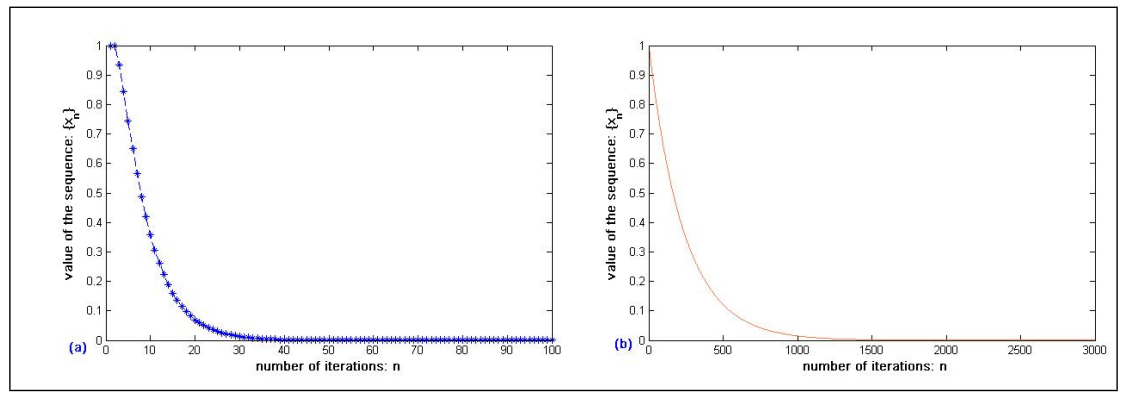

Figure 1: The convergence process of the sequence $\left\{x_{n}\right\}$ with different $\left\{\alpha_{n}\right\}:(a) \alpha_{n}=\frac{1}{n} ;(b) \alpha_{n}=\frac{9}{10}$.

From Figure 1 above, we see that by using the algorithm (5.4), the sequence $\left\{x_{n}\right\}$ converges to the common fixed point 0 for the different parametric sequence $\left\{\alpha_{n}\right\}$.

Table 1: Partial values of the sequence $\left\{x_{n}\right\}$ in the experiment.

\begin{tabular}{lcc}
\hline $\mathrm{n}$ & $\alpha_{\mathrm{n}}=\frac{1}{\mathrm{n}}$ & $\alpha_{\mathrm{n}}=\frac{9}{10}$ \\
\hline 0 & 1.0000 & 1.0000 \\
5 & 0.6513 & 0.9785 \\
10 & 0.3068 & 0.9575 \\
15 & 0.1362 & 0.9370 \\
20 & 0.0589 & 0.9170 \\
30 & 0.0106 & 0.8782 \\
40 & 0.0018 & 0.8412 \\
50 & 0.0003 & 0.8059 \\
60 & 0.0001 & 0.7721 \\
61 & 0.0000 & 0.0002 \\
100 & 0.0000 & 0.6511 \\
500 & 0.0000 & 0.1215 \\
1000 & 0.0000 & 0.0151 \\
2000 & 0.0000 & 0.0002 \\
2103 & 0.0000 & 0.0002 \\
2104 & 0.0000 & 0.0001 \\
2366 & 0.0000 & 0.0001 \\
2367 & 0.0000 & 0.0000 \\
\hline
\end{tabular}

Some values of the sequence $\left\{x_{n}\right\}$ in the numerical experiments of Figure 1 are shown on Table 1 . Table 1 clearly indicates that the different parametric sequence $\left\{\alpha_{n}\right\}$ affects on the convergence rate of the sequence $\left\{x_{n}\right\}$. In a word, the results of numerical simulations demonstrate that the algorithm of Theorem 3.1 is effective, realizable, and convergent.

\section{Acknowledgment}

The authors would like to thank the referees for their valuable comments and suggestions which improved the original submission of this paper. This work was supported by the National Natural Science Foundation of China (61603227, 61573218), the Shandong Provincial Natural Science Foundation (ZR2015AL001) and the Project of Shandong Province Higher Educational Science and Technology Program (J15LI51).

\section{References}

[1] Y. I. Alber, Metric and generalized projection operators in Banach spaces: properties and applications, Theory and applications of nonlinear operators of accretive and monotone type, Lecture Notes in Pure and Appl. Math., Dekker, New York, 178 (1996), 15-50. 1 
[2] H. H. Bauschke, J. M. Borwein, P. L. Combettes, Essential smoothness, essential strict convexity, and Legendre functions in Banach spaces, Commun. Contemp. Math., 3 (2001), 615-647. 2

[3] B. A. Bin Dehaish, A. Latif, H. O. Bakodah, X.-L. Qin, A regularization projection algorithm for various problems with nonlinear mappings in Hilbert spaces, J. Inequal. Appl., 2015 (2015), 14 pages. 1

[4] B. A. Bin Dehaish, X.-L. Qin, A. Latif, H. O. Bakodah, Weak and strong convergence of algorithms for the sum of two accretive operators with applications, J. Nonlinear Convex Anal., 16 (2015), 1321-1336. 1

[5] D. Butnariu, A. N. Iusem, Totally convex functions for fixed points computation and infinite dimensional optimization, Applied Optimization, Kluwer Academic Publishers, Dordrecht, (2000). 2.2

[6] D. Butnariu, E. Resmerita, Bregman distances, totally convex functions, and a method for solving operator equations in Banach spaces, Abstr. Appl. Anal., 2006 (2006), 39 pages. 2.1, 2

[7] G. Cai, C. S. Hu, Strong convergence theorems of modified Ishikawa iterative process with errors for an infinite family of strict pseudo-contractions, Nonlinear Anal., 71 (2009), 6044-6053. 1

[8] Y. Censor, A. Lent, An iterative row-action method for interval convex programming, J. Optim. Theory Appl., 34 (1981), 321-353. 2

[9] S. Y. Cho, W.-L. Li, S. M. Kang, Convergence analysis of an iterative algorithm for monotone operators, J. Inequal. Appl., 2013 (2013), 14 pages. 1

[10] S. Y. Cho, X.-L. Qin, On the strong convergence of an iterative process for asymptotically strict pseudocontractions and equilibrium problems, Appl. Math. Comput., 235 (2014), 430-438. 1

[11] Y. Hao, Some results on a modified Mann iterative scheme in a reflexive Banach space, Fixed Point Theory Appl., 2013 (2013), 14 pages. 1

[12] Y. Hao, S. Y. Cho, Fixed point iterations of a pair of hemirelatively nonexpansive mappings, Fixed Point Theory Appl., 2010 (2010), 14 pages. 1

[13] Y. Haugazeau, Sur les inéquations variationnelles et la minimisation de fonctionnelles convexes, Ph.D. Thesis, Université de Paris, Paris, (1968). 1

[14] J. K. Kim, Strong convergence theorems by hybrid projection methods for equilibrium problems and fixed point problems of the asymptotically quasi- $\phi$-nonexpansive mappings, Fixed Point Theory Appl., 2011 (2011), 15 pages. 1

[15] J. K. Kim, S. Y. Cho, X.-L. Qin, Some results on generalized equilibrium problems involving strictly pseudocontractive mappings, Acta Math. Sci. Ser. B Engl. Ed., 31 (2011), 2041-2057. 1

[16] S. Reich, S. Sabach, A strong convergence theorem for a proximal-type algorithm in reflexive Banach spaces, J. Nonlinear Convex Anal., 10 (2009), 471-485. 2, 2, 2.4

[17] S. Reich, S. Sabach, Two strong convergence theorems for a proximal method in reflexive Banach spaces, Numer. Funct. Anal. Optim., 31 (2010), 22-44. 2.3

[18] S. Reich, S. Sabach, Two strong convergence theorems for Bregman strongly nonexpansive operators in reflexive Banach spaces, Nonlinear Anal., 73 (2010), 122-135. 1, 2.7

[19] R. T. Rockafellar, Characterization of the subdifferentials of convex functions, Pacific J. Math., 17 (1966), 497-510. 4.3

[20] G. C. Ugwunnadi, B. Ali, I. Idris, M. S. Minjibir, Strong convergence theorem for quasi-Bregman strictly pseudocontractive mappings and equilibrium problems in Banach spaces, Fixed Point Theory Appl., 2014 (2014), 16 pages. 1, 4, 2.9, $2.10,2.11$

[21] Z.-M. Wang, Strong convergence theorems for Bregman quasi-strict pseudo-contractions in reflexive Banach spaces with applications, Fixed Point Theory Appl., 2015 (2015), 17 pages. 1, 2.8

[22] Z.-M. Wang, X.-M. Zhang, Shrinking projection methods for systems of mixed variational inequalities of Browder type, systems of mixed equilibrium problems and fixed point problems, J. Nonlinear Funct. Anal., 2014 (2014), 25 pages. 1

[23] H. Zhang, Iterative processes for fixed points of nonexpansive mappings, Commun. Optim. Theory, 2013 (2013), 7 pages. 1 